\title{
Two birds, one stone: tadalafil is an effective treatment for men with both BPH-LUTS and ED
}

$\longrightarrow$

enign prostatic hyperplasia $(\mathrm{BPH})$ often leads to lower urinary tract symptoms (LUTS), including voiding, storage and postmicturition abnormalities. Erectile dysfunction (ED) also frequently coexists with $\mathrm{BPH}$-associated LUTS. Until recently, the treatment options available for men with BPH-LUTS included $\alpha_{1}$-adrenergic blockers ( $\alpha$-blockers) and $5 \alpha$ reductase inhibitors (5-ARIs). Both drug categories are effective, but can cause unpleasant adverse effects, particularly on sexual function. However, during the past few years, studies have suggested that phosphodiesterase type 5 (PDE5) inhibitors, in addition to their welldocumented efficacy in ED, can improve BPH-LUTS symptoms. Indeed, in October 2011, this drug was approved by the FDA for this dual-purpose use. Two new randomized, placebo-controlled clinical studies have supported this decision, and an animal-based study provides an insight to the possible mechanisms underlying the positive effects of tadalafil in patients with BPH-LUTS.

The first study, by a German group led by Hartmut Porst, assessed the efficacy and safety of tadalafil in men with LUTS suggestive of BPH. All participants were aged $\geq 45$ years, with $\geq 6$ months duration of BPH-LUTS, and International Prostate Symptom Score (IPSS) $\geq 13$ at baseline. Patients received either once-daily oral tadalafil $5 \mathrm{mg}(n=161)$ or placebo $(n=164)$ for 12 weeks. The main outcome measures included changes in IPSS, BPH Impact Index (BII) score, Patient and Clinician Global Impression of Improvement (PGII and CGII) scores, and-in sexually active participants with both BPH-LUTS and ED-changes in the International Index of Erectile Function-Erectile Function domain (IEFF-EF).

Patients in the tadalafil group showed significantly greater improvement in IPSS scores after 12 weeks compared to the placebo group ( -5.6 versus -3.6 ,

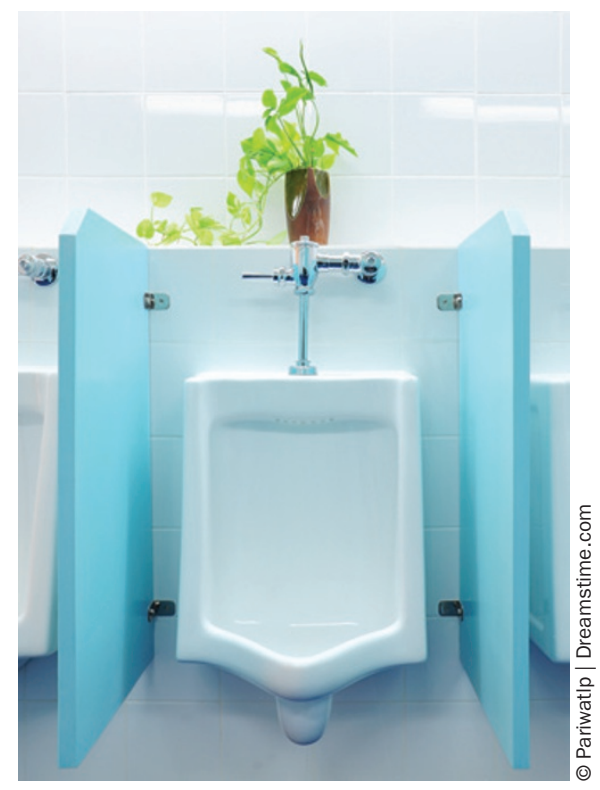

$P=0.004$ ), with this superiority becoming apparent after just 1 week and significant after 4 weeks. IPSS storage and voiding subscores were also improved to a significantly greater extent by tadalafil compared to placebo. A similar story was recorded for BII scores. The distribution of PGII and CGII scores in the tadalafil group indicated a greater feeling of improvement of urinary symptoms by both patients and clinicians. As would be expected, IEFF-EF scores at 12 weeks were significantly better in men receiving tadalafil. Only two serious adverse events were reported in the tadalafil group, one of which was unlikely to be associated with treatment. The most common adverse effects were back pain and headache.

The second study, by Russell Egerdie and colleagues, assessed the use of tadalafil in sexually active patients with coexisting BPH-LUTS and ED. Participants received 12 weeks' treatment with tadalafil $2.5 \mathrm{mg}$ $(n=198)$, tadalafil $5 \mathrm{mg}(n=208)$ or placebo $(n=200)$. Inclusion criteria were essentially the same as those of the study by Porst et al. with the addition of ED duration $\geq 3$ months.
Both tadalafil doses significantly improved IIEF-EF scores compared to placebo. Only patients in the tadalafil $5 \mathrm{mg}$ group showed significantly greater improvements in IPSS (including voiding and storage subscores), BII scores and Sexual Encounter Profile (SEP) question 3 scores compared to placebo. The adverse effect profile was very similar to that of the study by Porst and colleagues: predominantly headaches and back pain, with only a very low occurrence of serious adverse events.

Both studies showed significant improvements in BPH-LUTS and ED in patients treated with once-daily oral tadalafil $5 \mathrm{mg}$. But by what mechanism does tadalafil lead to improvements in BPH-LUTS? Morelli et al. examined the effects of tadalafil on human lower urinary tract tissues and in spontaneously hypertensive rats. They found that PDE5 was highly expressed-at levels comparable to the corpora cavernosa-in human vesicular-deferential-artery tissue, particularly in the smooth muscle bundles and endothelial cells, and that it could be successfully inhibited by tadalafil in vitro. Tadalafil treatment in hypertensive rats caused significant increases in prostate tissue oxygenation, implicating this as a possible mechanism by which PDE5 inhibition leads to improved BPH-LUTS.

Nick Warde

Original articles Egerdie, R. B. et al. Tadalafil 2.5 or $5 \mathrm{mg}$
administered once daily for 12 weeks in men with both
erectile dysfunction and signs and symptoms of benign
prostatic hyperplasia: results of a randomized, placebo-
controlled, double-blind study. J. Sex. Med. doi:10.1111/
j.1743-6109.2011.02504.x | Porst, H. et al. Efficacy and
safety of tadalafil once daily in the treatment of men with
lower urinary tract symptoms suggestive of benign prostatic
hyperplasia: results of an international randomized, double-
blind, placebo-controlled trial. Eur. Urol. $60,1105-1113$
(2011) | Morelli, A. et al. Phosphodiesterase type 5
expression in human and rat lower urinary tract tissues
and the effect of tadalafil on prostate gland oxygenation
in spontaneously hypertensive rats. J. Sex. Med. 8,
$2746-2760$ (2011)

